\title{
How long to keep the surgical drains- looking for evidence
}

\author{
Abstract \\ Objective: Closed suction drainage is the standard of care for head and neck surgical \\ wounds. \\ The aim of study is to determine the effective duration of surgical drains in different types \\ of Head and Neck procedures.
}

Study design: Prospective randomized trial in a tertiary referral center.

Methods: A total of 153 patients were divided into five groups. The study included patients with Head and Neck malignancies undergoing surgery. The patients in each group were assigned to two groups (Day 2 and Day 5) and drain output was measured.

Results: The total drainage was found to be more in laryngectomy group followed by neck dissection group, then by primary lesion excision with neck dissection followed by thyroidectomy group and lowest in Parotidectomy group. It was found that maximum drainage occurred in first 24hours after surgery, with subsequent decrease in drainage volume in all the groups.

Conclusion: It is safe to remove the surgical drain after 48hours of major Head and Neck surgery. There were minimum drain related side effects in patients who had their drain removed in 48 hours. There was a positive correlation between intraoperative blood loss and postoperative drainage in all groups. The drainage fluid after Major Head and Neck surgery is transudative in nature.
Volume 2 Issue 3 - 2015

\author{
Naresh K Panda, Monika Sood, Darwin \\ Kaushal, Jaimanti Bakshi, Roshan K Verma \\ Department of Otolaryngology, Post Graduate Institute of \\ Medical Education and Research, India
}

\begin{abstract}
Correspondence: Naresh K Panda, Department of Otolaryngology, Post Graduate Institute of Medical Education and Research, PGIMER, Chandigarh, India, Tel +91-17227|4021, Fax+9| I72274440I, Email npanda59@yahoo.co.in
\end{abstract}

Received: January 29, 2015 | Published: February 25, 2015

Keywords: head and neck surgery, drainage, postoperative period

\section{Introduction}

Head and neck operations are associated with clinically significant postoperative morbidities such as haematoma, seroma, wound infection and skin flap complications. ${ }^{1}$ Closed suction drainage is the standard of care for Head and Neck surgical wounds. The purpose of drains is to obliterate dead space and to reduce seroma and hematoma formation, thus improving skin apposition and wound healing, particularly when extensive undermining has been done. Additionally, it helps to characterize fluid for early identification of anastomotic leakage. ${ }^{2,3}$

Various factors regarding decisions to routinely use drains include the surgeon's training, experience and personal preference. ${ }^{4}$ However, the use of drains is not without consequences as they require a high level of maintenance, cause discomfort, and serve as potential route for infection when drainage is prolonged. ${ }^{5}$

The complications of the drain include pain, post operative infection and increased scarring. ${ }^{4}$ The timing of drain removal may have a significant influence on the duration of hospitalization and hence the cost of care. ${ }^{5}$ The optimal time to remove drains has not been well defined. A routine practice is to measure the drainage daily and it is removed when drainage falls below $25 \mathrm{ml}$ in last 24hours. ${ }^{6}$

This study is aimed to determine patterns of suction and drainage in elective Head and Neck surgery. The objective was to know the effective duration of surgical drains in different types of Head and Neck procedures.

\section{Materials and methods}

The aim of the study was to establish guidelines for determining the duration of surgical drains in different types of head and neck procedures. This study was a prospective randomized trial, which was conducted in the Department of Otolaryngology and Head and Neck surgery, Post Graduate Institute of Medical Education and Research, Chandigarh India over a period of one and a half year from Jan 2011 to June 2012.The study patients were in the age range of 15 to 75 years of both sexes .A total of 153 patients are included. The study included patients with Head and Neck malignancies undergoing surgery. All the procedures were performed by three surgeons (NKP, $\mathrm{JB}, \mathrm{RKV})$ who have almost similar experience in performing Head and Neck Surgery.

The proposed study was approved by the Ethics Committee of the Post graduate Institute of Medical Education and Research Chandigarh India.

Group T: Included patients of thyroid surgery, who underwent total thyroidectomy, hemithyroidectomy/ lobectomies without lateral neck dissection

Group N: Included patients undergoing radical, modified radical and selective neck dissections.

Group P: Included patients with superficial and total parotidectomy.

Group L: Included patients undergoing wide field Laryngectomy

Group NP: Included patients with resection of primary lesion with neck dissection. 
The patients in each group were assigned to two groups (D2 and D5) based on the computerized randomization table. In Day 2 group, the drain was removed on the $2^{\text {nd }}$ post operative day and in Day 5 group, the drain was removed on $5^{\text {th }}$ post operative day. The patients in both arms underwent clinical examination in addition to the basic hematological and biochemical investigations.

The patients were divided into five groups. Groups T, P and NP had minimum of 30 subjects each and group $\mathrm{N}$ and $\mathrm{L}$ had minimum 20 subjects. Patients with age of more than 14years and less than 75years were included. Patients with revision surgery, age more than 75 years or less than 14years, those undergoing any composite resection, any reconstruction and with bleeding diathesis were excluded from the study.

At the end of surgery every patient had a single radivac (negative suction drain in situ). These patients were observed daily. The total intraoperative blood loss, amount and duration of postoperative drainage were noted in all the patients. Finally the total drainage in 2 days or 5 days was calculated according to group to which patient belongs .Immediate complications of drain placement were also watched for as for such as e. g signs of inflammation, fever, local site pain or tenderness, evidence of seroma formation, hemorrhage, hematoma and post operative wound complication. Biochemical analysis of drain fluid was also performed.

Prophylactic antibiotics ceftriaxone or amoxicillin plus clavulinic acid were administered at the time of induction and were continued till removal of closed suction drain as per the existing protocol of the Department.

The patient data including age, gender, predisposing factors, anticoagulation use, and history of hypertension were recorded. Additional variables in the analysis were estimated intraoperative blood loss, blood transfusion during surgery, type of surgery, length of hospital stay and final pathology.

The follow up was done after 1 week of discharge to look for wound dehiscence, scarring and necrosis. The statistical analysis was carried out using Statistical Package for Social Sciences (SPSS Inc., Chicago, IL, version 15.0 for Windows). All quantitative variables e .g age, total duration, blood loss and biochemical analysis of drain fluid and blood was estimated using measures of central location mean, median and measures of dispersion like standard deviation and standard error. Normality of data was checked by measures of skewness and Kolmogorov Smirnov tests of normality.

\section{Results}

A total of 153 patients were evaluated who had undergone different types of Head and Neck surgical procedures (Table 1). 77 patients out of 153 had their drains removed on day 2 and 76 patients on day 5 .

The total drainage in each group is shown in Table 2. It was seen that total drainage was maximum in group $\mathrm{L}$ followed by $\mathrm{N}$ followed by NP followed by $\mathrm{P}$ and lastly in group T. The difference in total drainage in each group was statistically not significant with $p$ value 0.111 .

Table 3 shows the analysis of drainage collection in all groups in relation to duration after surgery. The drainage in all groups gradually reduced in volume from day 2 today 5.The drainage volume was compared between day 2 and day 5 in each group. . It was seen that maximum drainage occurred in first 48 hours after surgery with subsequent decrease in volume. There was no significant change in the volume of drainage after 48 hours in subsequent days i.e. day 3 , day 4 , and day 5 .

Table I Showing distribution of patients into different groups

\begin{tabular}{|c|c|c|}
\hline Group & Number of Patients & $\begin{array}{l}\text { Total number of } \\
\text { Patients }\end{array}$ \\
\hline Group T & $\begin{array}{l}\text { Hemithyroidectomy - } 31 \\
\text { Near total thyroidectomy- } 4 \\
\text { Total thyroidectomy-15 }\end{array}$ & 50 \\
\hline Group N & $\begin{array}{l}\text { RND- } 12 \\
\text { MND-5 } \\
\text { SOND-4 }\end{array}$ & 21 \\
\hline Group P & $\begin{array}{l}\text { Superficial parotidectomy-28 } \\
\text { Total conservative } \\
\text { parotidectomy- } 3\end{array}$ & 31 \\
\hline Group NP & $\begin{array}{l}\text { Neck dissection with primary } \\
\text { in upper aero digestive tract. }\end{array}$ & 30 \\
\hline Group L & Total Laryngectomy & 21 \\
\hline $\begin{array}{l}\text { Total number } \\
\text { of patients } n=\end{array}$ & & 153 \\
\hline
\end{tabular}

RND, radical neck dissection; mnd, modified neck dissection; sond, supra omohyoid neck dissection

Table 2 Showing total drainage in each group

\begin{tabular}{lll}
\hline Group & $\begin{array}{l}\text { Mean of Total } \\
\text { Drainage }(\mathrm{ml})\end{array}$ & Range(ml) \\
\hline Group T & 121.40 & $20-300$ \\
Group N & 148.81 & $60-500$ \\
Group P & 80.97 & $20-250$ \\
Group NP & 134.87 & $35-370$ \\
Group L & 153 & $35-320$ \\
\hline
\end{tabular}

Table 3 Showing median of total drainage through day I to day 5

\begin{tabular}{llllll}
\hline Group & DI & D2 & D3 & D4 & D5 \\
\hline Group T & 50 & 30 & 20 & 10 & 10 \\
Group P & 40 & 25 & 17.5 & 10 & 10 \\
Group N & 80 & 50 & 40 & 20 & 10 \\
Group NP & 65 & 45 & 30 & 12.5 & 10 \\
Group L & 100 & 40 & 20 & 10 & 10 \\
\hline
\end{tabular}

There was significant difference in mean of total drainage on day 2 (D2 )and day 5 (D5) with a significant difference in neck dissection, neck dissection with primary, Parotidectomy group and Thyroidectomy group with $\mathrm{p}$ value in group L 0.013, in group N .053, group NP .000, group P .000 and in group T .004 (Table 4).

We also correlated the intraoperative blood loss with rate of total drainage in various groups and found that there was a positive correlation between intraoperative hemorrhage and amount of surgical drainage. The $\mathrm{p}$ value in various groups were group L 0.000 , group $\mathrm{N}$ 0.015 , in group NP 0.012 , group T 0.000 and in group P 0.017 (Figure $1)$. 
Table 4 Showing results of biochemical analysis of drain fluid

\begin{tabular}{|c|c|c|c|c|c|c|}
\hline \multirow[t]{3}{*}{ Parameters } & \multicolumn{3}{|c|}{ Day 2 (D2) } & \multicolumn{3}{|l|}{ Day 5 (D5) } \\
\hline & \multicolumn{3}{|c|}{ Mean Value } & \multicolumn{3}{|l|}{ Mean Value } \\
\hline & Drain & Serum & P Value & Drain Fluid & Serum & PValue \\
\hline Sodium & 138.47 & 137.05 & .037 & 134.7 & 136.05 & .147 \\
\hline Urea & 29.7 & 34.86 & 0 & 30.6 & 33.04 & .267 \\
\hline Creatinine & .62 & .74 & 0 & .69 & .77 & .041 \\
\hline Cholesterol & 49.07 & 49.07 & 0 & 48.33 & 48.33 & 0 \\
\hline Triglyceride & 40.41 & 128 & 0 & 39.7 & 132 & 0 \\
\hline HDL & 29.85 & 44.4 & 0 & 26.88 & 43 & 0 \\
\hline LDL & 24.6 & 83 & 0 & 26.26 & 75.5 & 0 \\
\hline
\end{tabular}

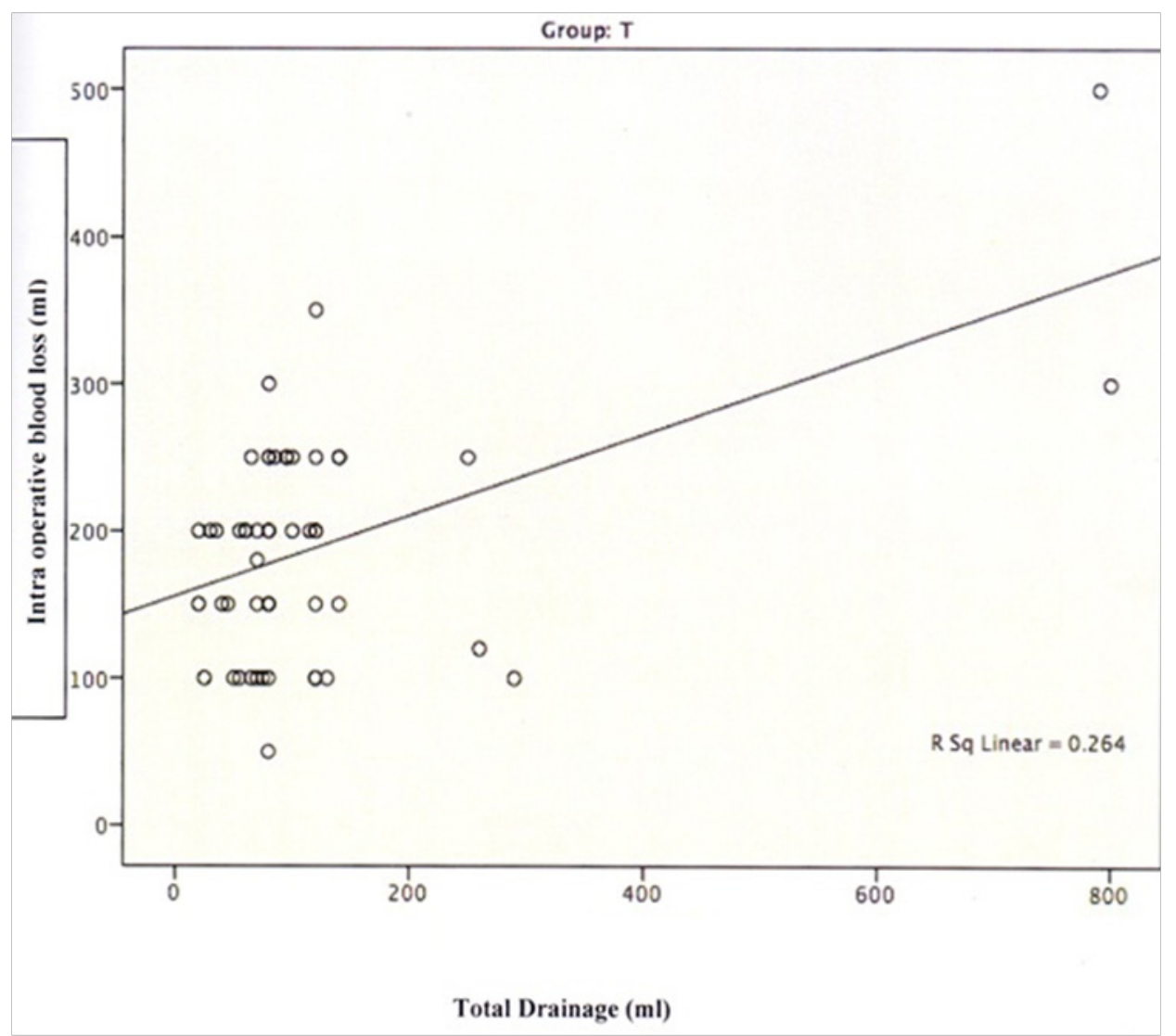

Figure I Showing relationship between intraoperative blood loss and post operative drainage in Thyroidectomy group. 
The biochemical analysis of drain fluid and blood were done on day 2 (D2) and day 5(D5) to compare differences in composition of drain fluid and blood. We found that there is a significant difference in drain fluid composition in the following parameters viz. protein, albumin, cholesterol, triglyceride, HDL, LDL in comparison to serum on day 2 as well as on day 5 .But no significant difference could be found in sodium, potassium, urea, creatinine of drain fluid in comparison to blood on day 2 (D2) and day 5 (D5).

We had drain related complications in four patients. Seroma was seen in two radical neck dissection patients and one total thyroidectomy patient and one in patient who underwent superficial parotidectomy. The seromas were small and resolved spontaneously. All these complications were encountered in day 2 (D2) group.

\section{Discussion}

The removal of neck drains after major Head and Neck surgery has remained a matter of debate. The timing of removal of neck drains vary among surgeons and Institutions. Firm guidelines to this effect are lacking in the literature.

In this prospective randomized study, we evaluated 153 patients who underwent various Head and Neck procedures. It was seen that the total drainage was maximum in-group $\mathrm{L}$ followed by group $\mathrm{N}$, followed by group NP followed by group T and lowest in-group P. The difference in total drainage amongst these groups was not significant.

We also compared the total drainage volume on day 2 and day 5 in all the groups and noticed that there was significant difference in mean of total drainage on day 2 and day 5 in all the groups. It was found that maximum drainage occurred in first 48 hours after surgery, with subsequent decrease in drainage volume in all groups. It is noteworthy to mention that this happened irrespective of operative surgeon who had similar experience and expertise.

Another fact which we noted was that there was minimal drainage collection of $10 \mathrm{ml} /$ day in all the groups on day 3 , day 4 and day 5 it could thus be deduced that drainage occurring after day 2 i.e. on day 3,4 , and 5 could be due to drain induced fluid exudation and not because of the surgical procedure. On reviewing the available English literature, there are no previous reports of similar findings.

Our study has shown evidence that it is prudent to remove the surgical drain after 48 hours of surgery. We measured drainage amount every 24 hourly and found highest drainage volume occurring in first 48 hours after surgery. There was significant decrease in drainage in subsequent days i.e. day3, day4, day 5 in all the groups. Hence, keeping the drain longer does not serve much purpose and may in fact harm the patients since longer the drain remain, more are the chances of further exudation and seroma formation. Presence of drain for long duration may in fact encourage further drainage by stimulating tissue reaction or by creating suction effect. Vacuum created by the negative suction of drain may prevent lymphatics from sealing off and thus cause increase in seroma formation and drainage and possible infection. Suffice to say that early drain removal prevents complications, decreases hospitalization and cost effectiveness. Similar observations have been reported by Amir et al., ${ }^{6}$ and Guyot et al.

Harris et al., 5 in a recent publication have reported study of 47 patients who had undergone Head and Neck surgery to determine safety and cost effectiveness of removing the drain when amount of drainage over a $24 \mathrm{hr}$ period fell to $<50 \mathrm{ml}$ or less. The authors reported that drain removal according to this protocol was successful in $91 \%$ cases, as only four patients ( $9 \%$ ) developed seroma following drain removal.

Our study on the contrary has more number of subjects $(n=153)$. The mean of total drainage on day 2 in all groups was $<50 \mathrm{ml}$. We removed drain in 77 patients on day 2. Only four patients out of seventy-seven patients (5\%) developed a seroma. All the remaining 73 patients had an uneventful outcome. This further reflects the safety of removal of surgical drains after 48 hours of surgery. The four patients with seroma were small ones and had no serious consequences.

We also analyzed the duration of keeping the surgical drains in various subgroups of major Head and Neck surgery patients. We found that in all of our 50 patients who underwent thyroidectomy (total thyroidectomy $n=19$, hemithyroidectomy $n=31$ ) the maximum drainage occurred in first 48 hours of surgery. We saw seroma in only one patient. It can thus be concluded that keeping the drain for 48 hours in patients undergoing thyroidectomy does not compromise surgical safety. On the contrary, many authors have reported no use of drain after thyroid surgery Lee ${ }^{8}$, Khanna ${ }^{9}$, Morrissey. ${ }^{10}$

We also correlated the postoperative surgical drain collection with intra operative blood loss. The blood loss was considered significant if it was more than $5 \%$ of the total blood volume of the patient. We came across a positive correlation between intra operative blood loss and postoperative drainage in all groups (spear man correlation $=.352$ $\mathrm{p}$ value $=.000$ ) . This is relevant for the Surgeon as increased drainage in the post-operative period may at times warrant delayed removal of the surgical drain.

To the best of our knowledge studies on biochemical analysis of the drain fluid is not available in the literature. It is speculated that there are conditions in which we expect either transudative or exudative drain fluid. Immediate exudative fluid can be resultant of leaking of blood along with red cells and late exudative can be because of presence of pus cells and WBC, resulting from wound infection. Late exudation may also result from chyle leakage from the lymphatic channels, which leads to increase amount lipoproteins in the drain fluid.

We noticed that initially drain fluid was reddish in appearance due to presence of blood on day 1-2. After that it changes to serosanguinous and subsequently to serous on day $3-5$ in most of the cases. We also found that there was significant difference in the levels of the following parameters viz. protein, albumin, cholesterol, triglyceride, HDL and LDL in drain fluid when compared with the serum on day 2 as well as on day 5 . On the other hand we noticed that there was no significant difference in sodium, potassium, urea, and creatinine of drain fluid in comparison to serum on day 2 and day 5 . These suggests that only the low molecular weight analytes can pass through the surgical wound after 2days of surgery, thus making the drain fluid more transudative in nature.

When we checked whether there are any biochemical changes in drain fluid, we found that fluid was transudative on day 2 and on day 5. The explanation for this is a matter of conjecture. The process of wound healing after surgery resulted in less leakage of cells and cellular debris along with high molecular weight plasma protein and lipoprotein. Only water with small molecules like glucose, urea oxygen comes out of cells. As the healing process progresses, there is 
a decrease in the leakage of lipoproteins, making the drain fluid more transudative. The absence of exudative fluid indicates that there was neither any infection nor chyle leak present in any of the enrolled patients. This study is important due to the fact that it highlights evidence to show that it is safe to remove the surgical drains after 48hours of major Head and Neck surgery.

\section{Acknowledgments}

None.

\section{Conflicts of interest}

The authors declare that there are no conflicts of interest.

\section{Funding}

None.

\section{References}

1. Memon MA, Memon MI, Donohue JH. Abdominal drains: a brief historical review. Jr Med J. 2001;94(6):164-166.

2. Pearl ML, Rayburn WF. Choosing abdominal incision and closure techniques: a review. $J$ Reprod Med. 2004;49(8):662-670.
3. Grobmyer SR, Graham D, Brennan MF, et al. High-pressure gradients generated by closed suction surgical drainage system. Surg Infect. 2002;3(3):245-249.

4. Dunlap WW, Berg RL, Urquhart AC. Thyroid drains and post operative drainage. Otolaryngol Head Neck Surg. 2010;143(2):235-238.

5. Harris T, Doolarkhan Z, Faqan JJ. Timing of removal of neck drains following head and neck surgery. Ear Nose Throat J. 2011;90(4):186-189.

6. Amir J, Morar P, Belloso A. Post operative drainage in Head and neck surgery. Ann R Coll Surg Engl. 2010;92(8):651-654.

7. Guyot A, Layer G. MRSA-'bug-bear' of a surgical practice: Reducing the incidence of MRSA surgical site infection. Ann R Coll Surg Engl. 2006;88(2):222-223.

8. Lee SW, Choi EC, Lee YM, et al. Is lack of placement of drains after thyroidectomy with central neck dissection safe? A prospective, randomized study. Laryngoscope. 2006;116(9):1632-1635.

9. Khanna J, Mohil RS, Chintamani, et al. Is the routine drainage after surgery for thyroid necessary? A prospective randomized clinical study. BMC Surg. 2005;5:11

10. Morrissey AT, Chau J, Yunker WK, et al. Comparison of drain versus no drain thyroidectomy. Randomized prospective clinical trial. J Otolaryngol Head Neck surg. 2008;37(1):43-47. 